SNUTP 94-124

\title{
RADIATIVE NEUTRON-PROTON CAPTURE IN EFFECTIVE CHIRAL LAGRANGIANS
}

\author{
Tae-Sun Park and Dong-Pil Min \\ Department of Physics and Center for Theoretical Physics \\ Seoul National University, Seoul 151-742, Korea \\ Mannque Rho \\ Service de Physique Théorique, CEA Saclay \\ 91191 Gif-sur-Yvette Cedex, France
}

\begin{abstract}
We calculate the cross-section for the thermal $n+p \rightarrow d+\gamma$ process in chiral perturbation theory to next-to-next-to-leading order using heavy-fermion formalism. The exchange current correction is found to be $(4.5 \pm 0.3) \%$ in amplitude and the chiral perturbation at one-loop order gives the cross section $\sigma_{t h}^{n p}=(334 \pm 2) \mathrm{m} b$ which is in agreement with the experimental value $(334.2 \pm 0.5) \mathrm{m} b$. Together with the axial charge transitions, this provides a strong support for the power of chiral Lagrangians for nuclear physics.
\end{abstract}

PACS: 12.39.Fe 21.30.+y 21.10.Ky 25.40.Lw

Typeset using REVTEX 
One of the corner-stones of nuclear physics is the successful explanation in terms of exchange currents given two decades ago by Riska and Brown [1] of the $\sim 10 \%$ discrepancy between the experimental cross-section and the theoretical impulse approximation prediction for the process

$$
n+p \rightarrow d+\gamma
$$

at threshold. Riska and Brown computed, using a realistic hard-core wave function for the deuteron, the two one-pion-exchange diagrams initially suggested in 1947 by Villars [2] plus the $\omega$ and $\Delta$ resonance diagrams. That the dominant contributions to electroweak exchange currents could be gotten from current-algebra low-energy theorems was suggested by Chemtob and Rho [3] who gave a systematic rule for organizing the leading exchangecurrent diagrams effective at low energy and momentum. Although suspected since the Yukawa force was introduced, the work of Riska and Brown was the first unequivocal evidence for the role of mesons, in particular that of pions, in nuclear interactions. In this Letter, we show that the terms considered by Riska and Brown are a (main) part of the terms that figure in chiral perturbation theory to next-to-next-to-leading $\left(\mathrm{N}^{2} \mathrm{~L}\right)$ order and that when completed by the rest of the $\mathrm{N}^{2} \mathrm{~L}$ order terms, chiral perturbation theory scores an impressive success in nuclei.

In the modern understanding of $\mathrm{QCD}$, it is the spontaneous breaking of chiral symmetry associated with the light quarks that predominantly governs the structure of low-energy hadrons as well as the forces mediating between them. In fact, the full content of the gauge theory of strong interactions, QCD, can be expressed at low energy by a systematic chiral expansion starting with effective chiral Lagrangians [ [4. Stated more strongly, such an approach, known as chiral perturbation theory $(\chi P T)$, while reproducing the current algebra, is now considered to be exactly equivalent to QCD in long wavelength regime [5. Our paper reports the first quantitative chiral perturbation calculation of the fundamental

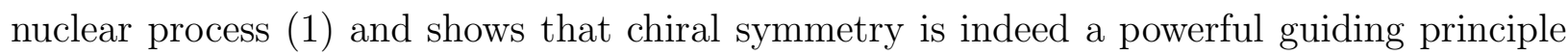
in nuclear dynamics, confirming the work of Riska and Brown [1] and the conjecture of Kubodera, Delorme and Rho [6].

Two recent developments provide a strong motivation for this work. The first is the work of Weinberg [7] and Ordóñez, Ray and van Kolck [8] on understanding nuclear forces from chiral Lagrangians. The second is the explanation by the present authors [9] of the enhanced axial-charge transitions in heavy nuclei in terms of exchange currents in chiral perturbation theory treated to the same chiral order as for nuclear forces.

In both cases cited above, one is limited to long wavelength processes, with the typical energy/momentum scale $Q$ much less than the chiral symmetry scale $\Lambda_{\chi} \sim m_{V} \sim 1 \mathrm{GeV}$. This is because $\chi P T$ is an expansion in $Q / \Lambda_{\chi}$ and its practical value lies where $Q / \Lambda_{\chi} \ll 1$. This entails a subtlety in applying $\chi P T$ to nuclear processes as we shall now specify, a 
feature absent in such "elementary processes" as $\pi \pi$ or $\pi N$ scattering for which much work with impressive success has been done [10,11.

The precise way $\chi P T$ can be applied in nuclear physics was explained in [9]. Here we sharpen the key arguments to bring home our thesis. For nuclear physics, where baryons as well as mesons are involved, the chiral expansion is made in heavy-fermion formalism 12,77 which allows a systematic expansion in derivative on pion fields, $\partial_{\pi} / \Lambda_{\chi}$ as well as on baryons fields, $\partial_{B} / X$ with $X=\Lambda_{\chi}$ or $m_{B}$ (baryon mass) and in $m_{\pi} / \Lambda_{\chi}$ where $m_{\pi}$ is the pseudo-Goldstone boson mass. The expansion is organized by the power $\nu$ in $Q^{\nu}$, given by Weinberg [7],

$$
\nu=4-N_{n}-2 C+2 L+\sum_{i} \Delta_{i}
$$

where $\Delta_{i}=d_{i}+\frac{1}{2} n_{i}-2$ and $N_{n}$ is the number of nucleons involved, $L$ the number of loops, $d_{i}\left(n_{i}\right)$ the number of derivatives or powers of pion mass (nucleon lines) that enter into the $i$ th vertex and $C$ the number of separated pieces of the Feynman graphs. In the presence of external fields (i.e, electroweak currents) which is what we want to study here, chiral invariance requires [13] that $\Delta_{i} \geq-1$. The $\chi P T$ in nuclear systems amounts then to compute Feynman diagrams involving external fields in the increasing power $\nu$ embedded inside the most general process describing the transition from the initial nuclear state to the final nuclear state with interactions taking place before and after the current insertion. This is essentially what was first suggested in [3] but on a somewhat ad hoc basis. What this means is that we are to take the most realistic nuclear wave functions and calculate the transition matrix elements with the $\chi P T$ graphs computed to the maximum possible order of chiral expansion. It is in this sense that the "counter terms" in the chiral expansion can be fixed from experiments. This point has been emphasized also by Weinberg [7] in his discussion of nuclear forces, in particular many-body forces. An important consequence of this strategy is then that only the current operators obtainable by $\chi P T$ are to be kept. This implies that (1) short-wavelength effects are to be "filtered out" and (2) n-body currents with $n>2$ are suppressed in the same sense that $n$-body forces are suppressed [7]. Of particular importance of the first implication is that when the currents are put in coordinate space, shorter-range interactions for $r_{12} \lesssim r_{c}$ where $r_{c}$ is the hard core radius cannot contribute in $\chi P T$. If the strategy is correct, the result should not sensitively depend on the precise value of $r_{c}$, within the relevant range for application in nuclei to the order considered, say, $\Lambda_{\chi}^{-1} \lesssim r_{c} \lesssim\left(2 m_{\pi}\right)^{-1}$. Note that this is roughly the range that has been successfully described in $\chi P T$ for the NN potential [8].

We now focus on the process (1) at thermal energy. The relevant operator is the isovector magnetic moment operator which we shall denote $\mu$. The vector current for the two-nucleon process consists of the one-body current $J_{(1)}^{\mu}$ (called "impulse current") and the two-body 
current $J_{(2)}^{\mu}$ (called "exchange current"). Although the time part of the leading order onebody current has $\nu=-3$ according to the counting rule (2), the space part - which is relevant to the $\mu$ - has $\nu=-2$, suppressed by a factor of order $\mathcal{O}\left(Q / m_{N}\right)$ for exactly the same reason as for the suppression of the time component of the single-particle axial-current [9]. The leading order two-body current has $\nu=-1$ with one-loop corrections entering at $\nu=+1$. Thus from the point of view of chiral expansion, the two-body current at one-loop order corresponds to next-to-next-to-leading $\left(\mathrm{N}^{2} \mathrm{~L}\right)$ order for the magnetic moment operator. This is the order that was computed in the case of the nuclear axial-charge transitions studied in Ref. [9] and that will be adopted for the vector current matrix element for the process (回).

To the $\mathrm{N}^{2} \mathrm{~L}$ order, the one-body current is the usual impulse isovector magnetic moment operator with the renormalized vertex i.e., $\mu_{(1)}=\frac{e}{4 m_{p}} \mu_{V} \sum_{i} \tau_{i}^{z} \sigma_{i}$ where $m_{p}$ is the proton mass and $\mu_{V} \equiv \mu_{p}-\mu_{n} \simeq 4.70589$. Now to the next-to-leading order (i.e., $O(Q)$ relative to the impulse), the two two-body currents Fig.1(a1) and (a2) contribute. With the renormalizations of the vertices and of the nucleon lines, they give the "tree two-body current" involving the renormalized (measured) constants $g_{A}=1.257$ and $f_{\pi}=93 \mathrm{MeV}$. The resulting operator will be denoted by $\mu_{(2) t r e e}$. We should mention that this is the same as what one gets from the corresponding "pair" and "pionic" currents given in [3] with the renormalized $\pi N N$ coupling constant $g_{\pi N N}$ replaced by $m_{N} g_{A} / f_{\pi}$ through the Goldberger-Treiman relation.

Going to the $\mathrm{N}^{2} \mathrm{~L}$ order (i.e., $O\left(Q^{3}\right)$ relative to the impulse), we encounter two classes of two-body currents: one-pion exchange with one-loop radiative corrections at the vertices and two-pion exchange. Part of the former class of diagrams renormalize the soft-pion exchange current to give the tree two-body current mentioned above. Besides this trivial correction, there is a nontrivial contribution coming from finite counter terms in the $\pi \mathcal{V} N N$ vertex in Fig.1(a1),

$$
\begin{aligned}
\mathcal{L}_{\mathrm{CT}} & =\frac{g_{A}^{3} c}{4 f_{\pi}^{2}} \bar{B} \epsilon^{\mu \nu \alpha \beta} v_{\mu} \Delta_{\nu}^{a} \Gamma_{\alpha \beta}^{a} B \\
& +\left[\frac{i g_{A} c^{\prime}}{4 f_{\pi}^{2}} \bar{B} \Delta_{\mu}^{a}\left(v^{\mu} v^{\nu}-g^{\mu \nu}-\frac{4}{3} S^{\mu} S^{\nu}\right)\left(\delta_{a b}-\frac{\tau_{a} \tau_{b}}{3}\right) \Gamma_{\nu \alpha}^{b} S^{\alpha} B+\text { h.c. }\right]
\end{aligned}
$$

where $\Gamma_{\mu \nu}=\frac{\tau_{a}}{2} \Gamma_{\mu \nu}^{a}=\partial_{\mu} \Gamma_{\nu}-\partial_{\nu} \Gamma_{\mu}+\left[\Gamma_{\mu}, \Gamma_{\nu}\right]$ and $\Gamma_{\mu}=\frac{\tau_{a}}{2} \Gamma_{\mu}^{a}\left(\Delta_{\mu}=\frac{\tau_{a}}{2} \Delta_{\mu}^{a}\right)$ is a vector (axial-vector) covariant combination of pion fields which contains either one derivative or an external gauge field. It can be shown that the counter-term constants $c$ and $c^{\prime}$ can be obtained by saturating with the resonances $\omega$ and $\Delta$ respectively,

$$
\begin{gathered}
\bar{c}_{\omega} \equiv \frac{g_{A}^{2} m_{\pi}^{2}}{f_{\pi}^{2}} c=\frac{g_{\omega}^{2} m_{\pi}^{2}}{8 \pi^{2} g_{A}\left(m_{\omega}^{2}-m_{\pi}^{2}\right)} \simeq 0.1021, \\
\bar{c}_{\Delta} \equiv \frac{2 m_{\pi}^{2}}{9 f_{\pi}^{2}} c^{\prime}=\frac{2 \mu_{T} \mathcal{C} m_{\pi}^{2}}{9 g_{A}\left(m_{\Delta}-m_{N}\right) m_{N}} \simeq 0.1667
\end{gathered}
$$


where $g_{\omega}$ is determined from the $\omega \rightarrow \pi \gamma$ decay, $g_{\omega}=17.55$, and the $N \Delta$ transition magnetic moment $\mu_{T}$ and the $\pi N \Delta$ coupling $\mathcal{C}$ come from the fit to the $\Delta$ properties as explained in [14], $\mathcal{C}=-1.73$ and $\mu_{T}=-7.7 \pm 0.5$. The resulting one-pion exchange two-body current is given by the two graphs Fig.1(a3) and (a4). We shall denote the corresponding magnetic moment operator by $\mu_{(2) 1 \pi}$. While these are again identical to what was obtained in [3], their interpretation is entirely novel and significant: chiral symmetry tells us that they constitute the complete (isovector) one-pion exchange current corrections to $\mu_{(2) t r e e}$. In particular, there are no finite chiral loop corrections to the one-pion-exchange operator. This is in contrast to the case of axial-charge transitions [9]. It seems reasonable therefore to lump all four terms of Fig.1a together and call them "generalized tree operators."

There are numerous diagrams of genuine loop character that can contribute in general kinematics to the two-pion-exchange two-body current. Fortunately things simplify drastically for the process (1) in heavy-fermion formalism, with only four graphs (b1), (b2), (b3) and (b4) of Fig.1 non-vanishing: Two-body currents involving four-Fermi interactions are zero-ranged in coordinate space and together with all other zero-range terms give zero contributions due to the short-range cut-off (or correlation) as described in detail in [9]. Now these four graphs - which appear at the same chiral order as the above "generalized tree operators" - have hitherto been unaccounted for in this form in the previous studies of exchange currents. (It is possible however that part or all of this may have been included in heavy-meson exchange graphs in phenomenological approaches.) After divergences are removed (we use dimensional regularization), the resulting magnetic moment operator takes the form

$$
\begin{array}{r}
\mu_{(2) 2 \pi}=\frac{e m_{\pi}^{3} g_{A}^{2}}{16 \pi^{2} f_{\pi}^{4}}\left(\frac{2}{3} T_{S}^{(-)}-T_{T}^{(-)}\right) \int_{1}^{\infty} d t t \sqrt{t^{2}-1} y_{1}\left(2 t x_{\pi}\right) \\
+\frac{e m_{\pi}^{3} g_{A}^{4}}{16 \pi^{2} f_{\pi}^{4}} \int_{1}^{\infty} d t\left\{\frac{2}{3} T_{S}^{(\times)} \frac{t^{3}}{\sqrt{t^{2}-1}}\left(y_{1}\left(2 t x_{\pi}\right)-\frac{1}{2} y_{0}\left(2 t x_{\pi}\right)\right)\right. \\
\left.-T_{T}^{(\times)}\left(t \sqrt{t^{2}-1} y_{1}\left(2 t x_{\pi}\right)-\frac{2 t^{3}-t}{\sqrt{t^{2}-1}} y_{2}\left(2 t x_{\pi}\right)\right)\right\}
\end{array}
$$

where $x_{\pi}=m_{\pi} r, y_{0}(x)=\frac{\mathrm{e}^{-x}}{4 \pi x}, y_{1}(x)=(1+x) y_{0}(x)$ and $y_{2}(x)=\left(1+\frac{3}{x}+\frac{3}{x^{2}}\right) y_{0}(x)$. The spin-isospin operators are defined by $T_{S}^{(\odot)}=\left(\tau_{1} \odot \tau_{2}\right)^{z}\left(\sigma_{1} \odot \sigma_{2}\right)$ and $T_{T}^{(\odot)}=\left(\tau_{1} \odot\right.$ $\left.\tau_{2}\right)^{z}\left[\hat{r} \hat{r} \cdot\left(\sigma_{1} \odot \sigma_{2}\right)-\frac{1}{3}\left(\sigma_{1} \odot \sigma_{2}\right)\right]$ for $\odot=-$ and $\times$.

The total magnetic moment operator to the $\mathrm{N}^{2} \mathrm{~L}$ order then is

$$
\mu=\mu_{(1)}+\mu_{(2) \text { tree }}+\mu_{(2) 1 \pi}+\mu_{(2) 2 \pi} .
$$

Let the corresponding matrix elements for the capture process be denoted by $M_{i}$. The we are specifically interested in the ratios $\delta_{\text {tree }} \equiv M_{(2) t r e e} / M_{(1)}, \delta_{1 \pi} \equiv M_{(2) 1 \pi} / M_{(1)}, \delta_{(2) 2 \pi} \equiv$ $M_{(2) 2 \pi} / M_{(1)}$ and $\delta_{2 B}$ which is the sum. 
We now describe the numerical results. For this, we should pick the most realistic twonucleon wave functions. We take the Argonne potential $v_{18}$ recently constructed by Wiringa, Stoks and Schiavilla [15]. This potential is fit to $1787 \mathrm{pp}$ and $2514 \mathrm{np}$ scattering data in the range $0-350 \mathrm{MeV}$ with an excellent $\chi^{2}$ of 1.09 and gives the deuteron properties - the asymptotic S-state normalization, $A_{S}$, the $D / S$ ratio, $\eta$, and the deuteron radius, $d_{d}-$ close to the experimental values. Electromagnetic properties also come out well, modulo exchange-current and relativistic corrections.

We use the physical values for masses and constants that appear in the theory. There are no unknown parameters except one that has to do with nuclear interactions at short distance, namely short-range cutoff $r_{c}$. Figure 2 summarizes the results of the calculation for a wide range of $r_{c}, 0<r_{c} \lesssim 0.7 \mathrm{fm}$ compared with the experiment [16]. We describe very briefly how this result comes about. Details will be given in a later publication [17.

The capture cross section is proportional to $a_{s}^{2}$ where $a_{s}$ is the ${ }^{1} S_{0} n p$ scattering length. The Argonne $v_{18}$ predicts $a_{s}^{t h}=-23.732 \mathrm{fm}$ in excellent agreement with the experimental value $a_{s}^{e x p}=-23.739 \pm 0.008 \mathrm{fm}$. The single-particle matrix element with this potential gives the impulse approximation cross section (given by $\mu_{(1)}$ of Eq.(6)) $\sigma_{i m p}=305.6 \mathrm{mb}$, about $9.6 \%$ less than the experimental value $\sigma_{\exp }=334.2 \pm 0.5 \mathrm{mb}$. It has no $r_{c}$ dependence. The two-body matrix elements computed with the same wave function does depend on $r_{c}$ but very weakly. The resulting total cross section is plotted in Fig.2a for the relevant range of $r_{c}$. (The $r_{c}$ dependence is incorporated in the radial integral by multiplying the integrand by $\theta\left(r-r_{c}\right)$.) Figure $2 \mathrm{~b}$ shows the contribution of each term in terms of the ratios $\delta_{(2)}$. We note that the "generalized tree" contributions dominate to the $\mathrm{N}^{2} \mathrm{~L}$ order, with only a small correction (less than $0.6 \%$ of the single-particle matrix element) coming from the genuine one-loop correction. This agrees with the "chiral filter" mechanism seen in the axial-charge transitions [9] and confirms the conjecture made in [6]. The intrinsic uncertainty associated with short-distance physics notwithstanding, the theoretical prediction $\sigma_{\chi P T}=334 \pm 3 \mathrm{mb}$ (where the theoretical error bar represents the dependence on the hard-core cut-off) is in remarkable agreement with the experiment, say, within less than $1 \%$ !

In conclusion, we discuss the meaning of the short-distance cut-off $r_{c}$ in $\chi P T$. As discussed in [9], the loop terms contain zero-range operators in coordinate space. In addition, four-Fermi counter terms in the chiral Lagrangian with unknown constants are also zeroranged. At higher chiral order, increasingly shorter-ranged operators would enter together with the zero-ranged ones. Now if we were able to compute nuclear interactions to all orders in chiral perturbation theory, the delta functions in the current would be naturally regularized and would cause no problem. Such a calculation of course is an impossible feat. The practical application of chiral perturbation theory is limited to low orders in the chiral expansion, so a cut-off would be needed to screen the interactions shorter-ranged than accessible by the chiral expansion adopted. Clearly such a calculation would be meaningful only 
if the dependence on the cut-off were weak. Our calculation here and also the one in [9] meet that criterion. In the present work, we find $r_{c} \simeq 0.5 \mathrm{fm}$ at which the theoretical prediction agrees exactly with the experiment indicating that at one-loop order, chiral perturbation theory is meaningful for processes taking place at internucleon distances $r \gtrsim 0.5 \mathrm{fm}$. We conjecture that to the next order, that is $O\left(Q^{4}\right)$ relative to the leading tree graphs Fig.1(a1) and (a2), the appropriate cut-off would be $r_{c} \sim 1 / m_{\omega} \sim 0.3 \mathrm{fm}$. It would be a challenge to quantify this heuristic reasoning.

\section{Acknowledgments}

The work of TSP and DPM was supported in part by the Korea Science and Engineering Foundation through Center for Theoretical Physics, Seoul National University and in part by the Korea Ministry of Education under the grant No.BSRI-94-2418. 


\section{REFERENCES}

[1] D.O. Riska and G.E. Brown, Phys. Lett. B38, 193 (1972).

[2] F. Villars, Helv. Phys. Acta 20, 476 (1947).

[3] M. Chemtob and M. Rho, Nucl. Phys. A163, 1 (1971).

[4] S. Weinberg, Physica (Amsterdam), 96A, 327 (1979).

[5] H. Leutwyler, Ann. Phys. (N.Y.) 235, 165 (1994).

[6] K. Kubodera, J. Delorme and M. Rho, Phys. Rev. Lett. 40, 755 (1978).

[7] S. Weinberg, Phys. Lett. B251, 288 (1990); Nucl. Phys. B363, 3 (1991); Phys. Lett. B295, 114 (1992).

[8] C. Ordóñez, L. Ray and U. van Kolck, Phys. Rev. Lett. 72, 1982 (1994).

[9] T.-S. Park, D.-P. Min and M. Rho, Phys. Repts. 233, 341 (1993); T.-S. Park, I.S. Towner and K. Kubodera, Nucl. Phys. A579, 381 (1994).

[10] J. Gasser and H. Leutwyler, Ann. Phys. (N.Y.) 158, 142 (1984); Nucl. Phys. B307, 763 (1988).

[11] U.-G. Meissner, Rep. Prog. Phys. 56, 903 (1993).

[12] E. Jenkins and A.V. Manohar, in Proc. of the Workshop on Effective Field Theories of the Standard Model, Dobogókó, Hungary, Aug. 1991, ed. U.-G. Meissner (World Scientific, Singapore, 1992).

[13] M. Rho, Phys. Rev. Lett. 66, 1275 (1991).

[14] E. Jenkins, M. Luke, A. V. Manohar and M. Savage, Phys. Lett. B302, 482 (1993).

[15] R.B. Wiringa, V.G.J. Stoks and R. Schiavilla, "An accurate nucleon-nucleon potential with charge independence breaking," nucl-th/9408016.

[16] A.E. Cox, S.A.R. Wynchank and C.H. Collie, Nucl. Phys. 74, 497 (1965).

[17] T.-S. Park, D.-P. Min and M. Rho, in preparation. 
FIGURES

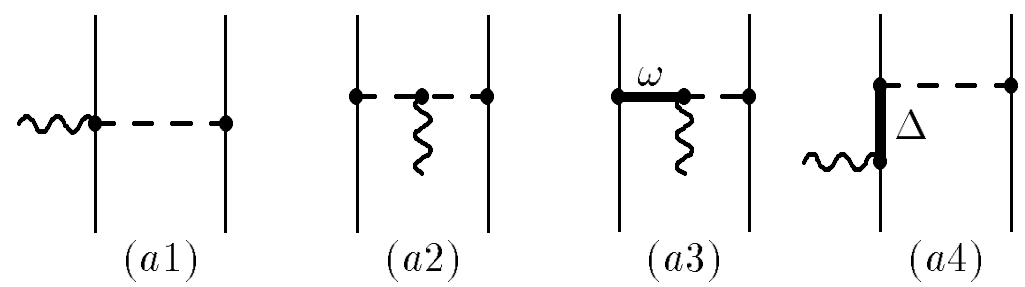

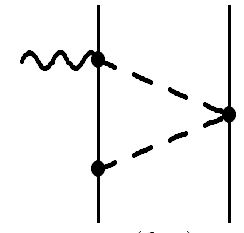

$(b 1)$

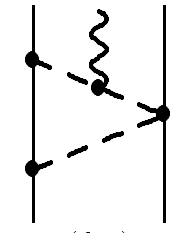

(b2)

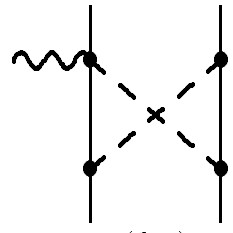

$(b 3)$

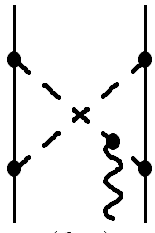

(b4)

FIG. 1. The Feynman graphs contributing to the two-body vector current for the process (1): (a) the "generalized tree graphs"; (b) two-pion exchange graphs. The current is depicted by the wiggly line, the pion by the broken line and the nucleon by the solid line. One-loop graphs figuring in $\pi \mathcal{V} N N$ vertex are entirely saturated at low photon energy by the two resonance-exchange-tree graphs $((a 3)$ and $(a 4))$.

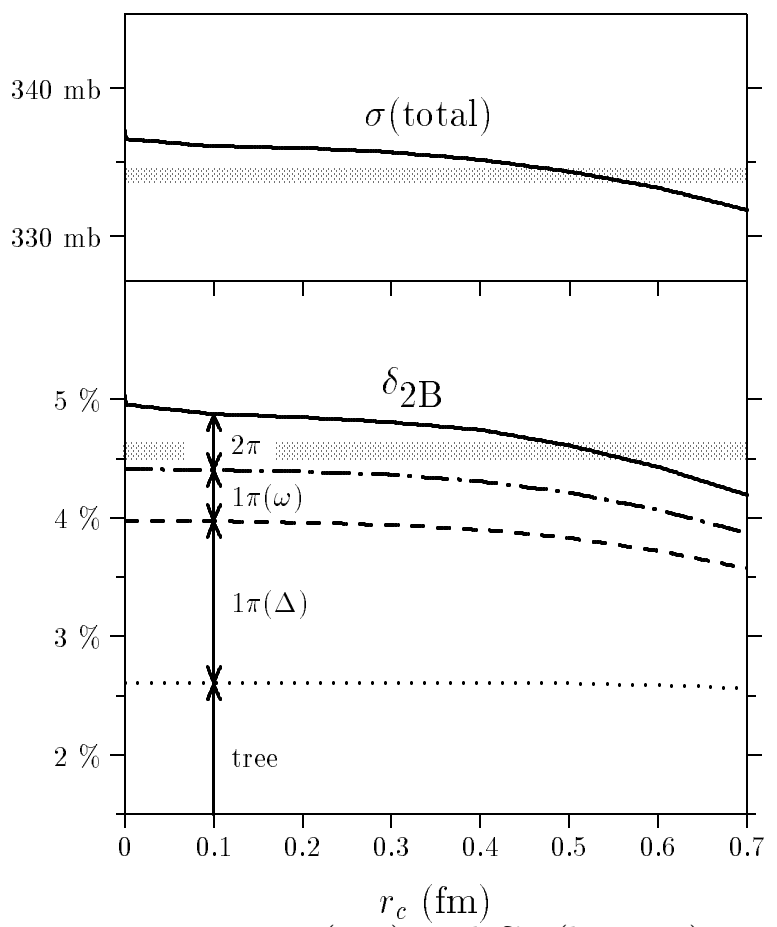

FIG. 2. Total capture cross section $\sigma_{\text {cap }}$ (top) and $\delta$ 's (bottom) vs. the cut-off $r_{c}$. The solid line represents the total contributions and the experimental values are given by the shaded band indicating the error bar. The dotted line gives $\delta_{\text {tree }}$, the dashed line $\delta_{\text {tree }}+\delta_{1 \pi}^{\Delta}$, the dot-dashed line $\delta_{\text {tree }}+\delta_{1 \pi}=\delta_{\text {tree }}+\delta_{1 \pi}^{\Delta}+\delta_{1 \pi}^{\omega}$ and the solid line the total ratio, $\delta_{2 \mathrm{~B}}$. 